\title{
Importance of Viscoelastic Property Measurement of a New Hydrogel for Health Care
}

\author{
Niladri Roy, Nabanita Saha, Takeshi Kitano and Petr Saha \\ Polymer Centre, Faculty of Technology, Tomas Bata University in Zlin, TGM 275, 76272 Zlin, \\ Czech Republic
}

\begin{abstract}
A simple technology based new hydrogel "PVP-CMC-BA" has been prepared by the scientists of Tomas Bata University in Zlin, Czech Republic. Its swelling property (in presence of water, human blood and different $\mathrm{pH}$ ), antimicrobial property (in presence of skin infection causing agents like: Staphylococcus aureus; bacteria and Candida albicans; fungi) and viscoelastic properties such as storage modulus $\left(G^{\prime}\right)$, loss modulus $\left(G^{\prime \prime}\right)$ and complex viscosity $\left(\eta^{*}\right)$ were investigated at room temperature $\left(25-28^{\circ} \mathrm{C}\right)$ which demonstrate that PVP-CMC-BA hydrogel is maintaining requisite properties for health care application, specially as a wound dressing material. The elasticity and antimicrobial property of PVP-CMC-BA is directly correlated with percentage of boric acid, an antiseptic agent. The consequential values of viscoelastic properties of the hydrogel (before drying) enable us to understand its specific flexible condition to apply on the surface of human body.
\end{abstract}

Keywords: Hydrogels, Boric acid, Swelling, Viscoelastic properties, Wound dressing. PACS: $87,83.85 . \mathrm{Cg}, 83.85 . \mathrm{Vb}$

\section{INTRODUCTION}

Viscoelasticity is described as a combination of viscous and elastic properties in a material with the relative contribution of each being dependent on time, temperature, stress and strain rate [1]. The viscoelastic properties of hydrogels correlate strongly with their microstructures and could provide useful information for modulating their performance characteristics [2]. Viscoelastic behavior of a matter is mainly explained by two theories: phenomenological theories use macroscopic stress-strain-time relationships and molecular theories provide evidence of viscoelastic behavior from molecular structure and conformation [3]. The viscoelastic behavior of gels is dependent on several factors, like composition and concentration of the dispersed materials and the lag time between preparation and measurement [4]. Till today, not much information is available regarding study of viscoelastic behavior of the wound dressing hydrogels. But it is earnestly important according to the application point of view. Wound dressing hydrogels are used on human and or animal body surfaces which are uneven and under movement. So, whenever hydrogels are applied on body surface (skin), they experience some strain. To withstand with such kind of external 
influences, hydrogels must be flexible in nature and the extent of flexibility can be determined by viscoelastic measurements.

Hydrogels are generally considered as two component systems, where one of the components is hydrophilic but water insoluble three dimensional polymer networks, and the second component is water [5]. Hydrogels can be successfully used for medical treatment for burns and cuts, because they have many interesting properties: immediate pain control, easy replacement, transparency, absorption and prevention of loss of body fluids, barrier against bacteria, good adhesion, easy handling, oxygen permeability, control of drug dosage etc. [6, 7]. That is why, present time dermatologists are taking interest to use hydrogel as dressing material for the patients to get relieve from the suffering of skin burns/wounds.

Keeping the view of present interest of hydrogel in medical field, in our laboratory at Tomas Bata University in Zlin, Czech Republic we developed PVP-CMC based hydrogel [8] with and without boric acid; an antiseptic cum antimicrobial agent. The preparation technique of PVP-CMC and PVP-CMC-BA hydrogel has already been filed for patent right [9]. Incorporation of boric acid (BA) provides antimicrobial property into the hydrogels to make it useful in medicinal purpose as well as for the quick healing of the wounds $[10,11]$ by inhibiting the microbial infections. This paper mainly focuses about the measurement of viscoelastic properties [12] of the PVP-CMC-BA hydrogels besides other associated fundamental properties, like liquid absorptivity and antimicrobial property.

\section{EXPERIMENTAL}

\section{Materials}

Polyvinylpyrrolidone K 30 (PVP: molecular weight 40,000), polyethylene glycol 3'000 (PEG: average molecular weight 3,015-3,685) and agar were supplied by Fluka, Switzerland; sodium carboxymethyl cellulose (CMC) was purchased from Sinopharm Chem. Reagent Co., Ltd., China; boric acid (BA) from Sigma-Aldrich, USA; glycerin obtained from Lachema Ltd., Czech Republic.

Among the components present in the resultant hydrogel PVP and CMC function as base polymers, PEG performs as healing agent, glycerin acts as a humectant, and agar is playing the role of gelling agent. Agar can form hydrogel on cooling the solution, because the gel is formed due to the formation of helices and its association.

\section{Preparation of PVP-CMC-BA Hydrogel}

The PVP-CMC-BA hydrogels were prepared by dissolving PVP $(0.2 \% \mathrm{w} / \mathrm{v}), \mathrm{CMC}$ $(0.8 \% \mathrm{w} / \mathrm{v})$, PEG $(1 \% \mathrm{w} / \mathrm{v})$, BA $(0.0-4.5 \% \mathrm{w} / \mathrm{v})$, agar $(2 \% \mathrm{w} / \mathrm{v})$ and glycerin $(1 \% \mathrm{v} / \mathrm{v})$ in distilled water. The polymer solutions were prepared in sealed bottles under $15 \mathrm{lbs}$ pressure and $120^{\circ} \mathrm{C}$ temperature for 20 minutes. Then, the liquids $(20 \mathrm{ml}$ each) were poured into Petri dishes $(80 \mathrm{~mm}$ diameter) and allowed to come at room temperature. Finally, smooth and whitish round hydrogels were obtained. PVP-CMC hydrogels were also been prepared following the same procedure as mentioned above, with all the same ingredients except BA. 


\section{Measurement of Viscoelastic Properties}

The viscoelastic behaviour of hydrogels was investigated by using a parallel plate rheometer (ARES; Rheometrics Scientific, USA) testing machine with an "RSI Orchestrator" software package. A $25 \mathrm{~mm}$ diameter parallel plate measuring geometry, with a gap of about 2-3 mm was used, employing at small strain amplitude $(1 \%)$ to maintain the measurements with the linear viscoelastic region (LVER). Dynamic frequency sweep tests were carried out at room temperature $\left(25-28^{\circ} \mathrm{C}\right)$ to observe the storage $\left(G^{\prime}\right)$ and loss moduli $\left(G^{\prime}\right)$ as a function of a wide range of angular frequencies $(\omega: 0.1-100 \mathrm{rad} / \mathrm{s})$. Influences of the PVP-CMC-BA composition on complex viscosity $\left(\eta^{*}\right)$ which is calculated by the Eq. 1 are also discussed.

$$
\eta^{*}=\left[\left(\frac{G^{\prime}}{\omega}\right)^{2}+\left(\frac{G^{\prime \prime}}{\omega}\right)^{2}\right]^{\frac{1}{2}}
$$

\section{Assay of Liquid Absorptivity}

The degree of swelling could be described as water or fluid absorptivity of the hydrogel. The dry films were obtained by drying the hydrogels at $40^{\circ} \mathrm{C}$ temperature until they reached constant weight. Small pieces $\left(1 \mathrm{~cm}^{2}\right)$ from the dry films were weighed and immersed in distilled water at room temperature. After the specified time intervals the water on the swollen gels was wiped out with tissue paper and the weight of specimens were determined. The same study was also performed in blood and in different buffer solutions $(\mathrm{pH}=1.7,4.0,7.0 \& 9.0)$.

The degree of swelling corresponds to the absorptivity of the material, which is defined by Eq. 2, where $W_{\mathrm{s}}$ and $W_{\mathrm{d}}$ are weights of swollen gel and dried gel, respectively.

$$
\operatorname{Absorptivity}(\%)=\left[\frac{W_{\mathrm{s}}-W_{\mathrm{d}}}{W_{\mathrm{d}}}\right] \cdot 100
$$

\section{Assay of Antimicrobial Property}

The antimicrobial property of these PVP-CMC-BA hydrogels has been investigated by agar diffusion method. Antimicrobial possessions of PVP-CMC-BA hydrogels were examined on the basis of dimension of inhibition zone generated in presence and absence of pathogenic bacterium Staphylococcus aureus and fungus like Candida albicans. The antibacterial assay was conducted in a sterile nutrient agar (2\%) medium which was inoculated with above mentioned bacterial strains under aseptic condition. After solidification of nutrient agar, a piece of hydrogel (15 $\mathrm{mm}$ diameter) was placed on the surface of medium. The testing plates were then incubated in a temperature controlled incubator at $37^{\circ} \mathrm{C}$ to notice the effect of PVP-CMC-BA hydrogels on the growth of individual bacteria. The antifungal assay was also conducted in the same manner as mentioned above, only the difference is that, malt extract agar (2\%) medium was used in spite of nutrient agar medium. 


\section{RESULTS AND DISCUSSION}

\section{Viscoelastic Properties}

Figure 1 shows the effect of angular frequency $(\omega)$ on storage modulus $\left(G^{\prime}\right)$ and loss modulus $\left(G^{\prime \prime}\right)$ for PVP-CMC-BA hydrogels with respect to BA concentration. In case of every sample, storage modulus is showing higher value than loss modulus and all the samples show the similar trend in behavior. All hydrogels show higher elastic property rather than viscous property. Moreover, elasticity gradually increases with the increasing concentration of boric acid in hydrogels. It may be because of the increasing quantity of BA which causes the formation of more crosslinked networks within the hydrogel. It can also be seen that the angular frequency does not affect so much on storage modulus and loss modulus, showing more or less similar to rubbery plateau behavior.

In Figure 2, complex viscosity $\left(\eta^{*}\right)$ represents how viscous and elastic properties influence on flow behaviour of the hydrogels. As we can see from Figure $1, G^{\prime}$ is always significantly higher than $G$ " for each samples, so we can say that the influences of storage modulus $\left(G^{\prime}\right)$ is predominant on the flow behaviour of the hydrogels. Complex viscosity also increases with the increase in BA concentration.

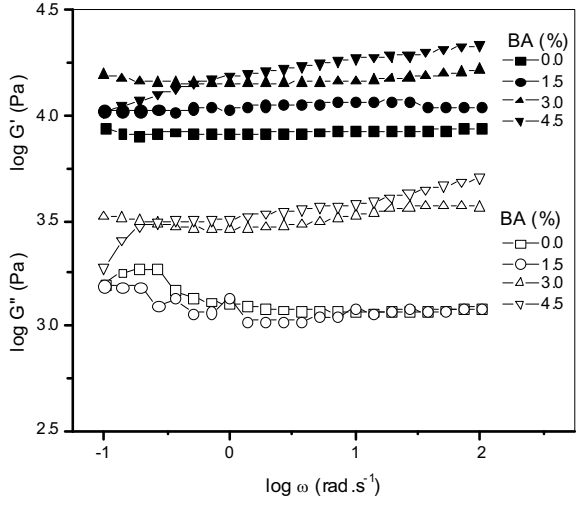

FIGURE 1. Effect of angular frequency $(\omega)$ on storage modulus $\left(G^{\prime}\right)$ and loss modulus $\left(G^{\prime \prime}\right)$ for hydrogels as a parameter of BA concentration.

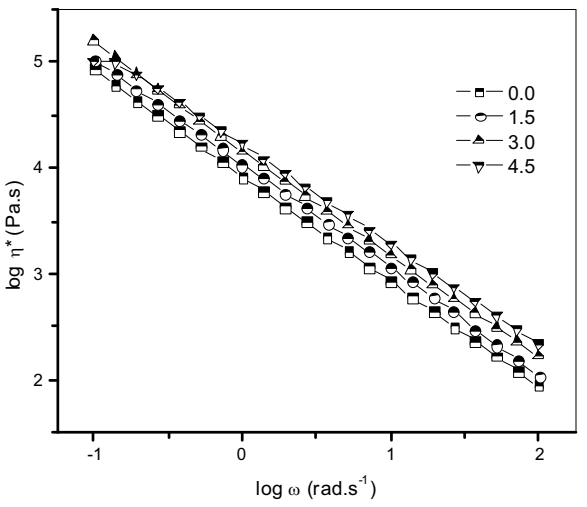

FIGURE 2. Effect of angular frequency $(\omega)$ on complex viscosity $\left(\eta^{*}\right)$ for hydrogels as a parameter of BA concentration.

Figure 3 presents the effect of different concentration of BA on the storage modulus $\left(G^{\prime}\right)$ and loss modulus $\left(G^{\prime \prime}\right)$ of the hydrogels in three different angular frequencies $(\omega$ $=0.37,3.7$ and $37 \mathrm{rad} / \mathrm{s}$ ). There is no significant change in the values of $G^{\prime}$ and $G$ ' ' on the application of different angular frequencies. These behavior mean that the hydrogels are well crosslinked, and then angular frequency can not influence much to change the internal structure of the crosslinked hydrogels. 


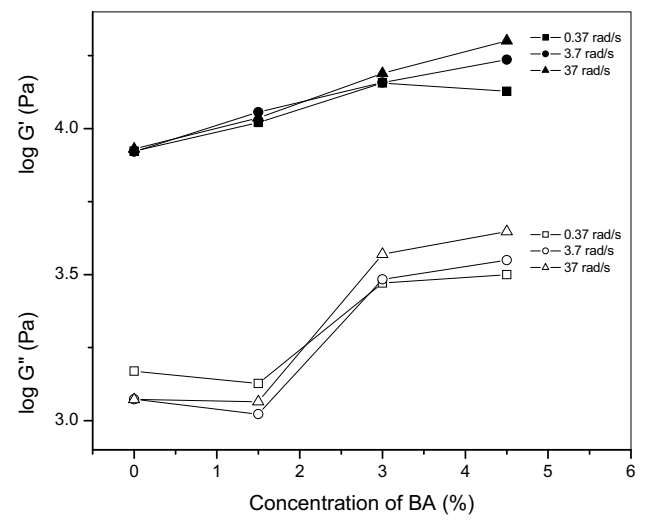

FIGURE 3. Effect of concentration of BA on storage modulus $\left(G^{\prime}\right)$ and loss modulus $\left(G^{\prime}\right.$ ') for hydrogels with respect to different angular frequencies $(\omega)$.

\section{Liquid Absorptivity}

Liquid absorptivity behaviour of the hydrogels was measured in distilled water (Figure 4), in blood (Figure 5) and in different $\mathrm{pH}$ (Figure 6). In water and blood PVP-CMC hydrogels without BA showing significantly higher swelling properties rather than other PVP-CMC-BA hydrogels. Degree of swelling decreasing gradually as the concentration of BA increases. That may be explained as the concentration of BA increases; it creates more cross links into the hydrogel, which causes lowering in percentage of swelling. From Figure 6 it can be seen that hydrogels behave differently in the buffer solutions of different $\mathrm{pH}$ values. They show higher swelling property in $\mathrm{pH} 4.0$ and 9.0 than $\mathrm{pH} 1.7$ and 7.0.

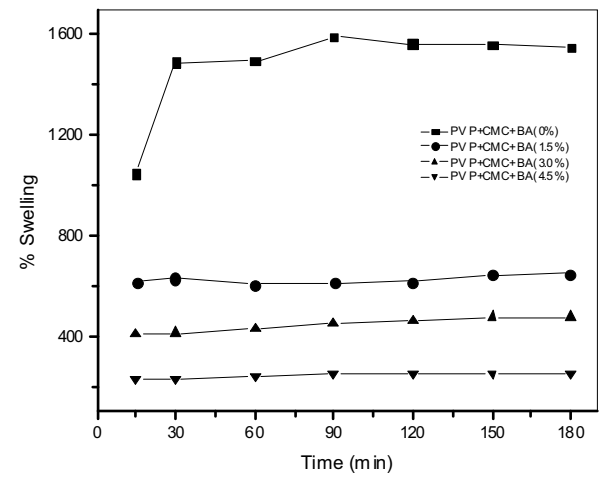

FIGURE 4. Swelling behavior of PVP-CMC-BA hydrogels in water.

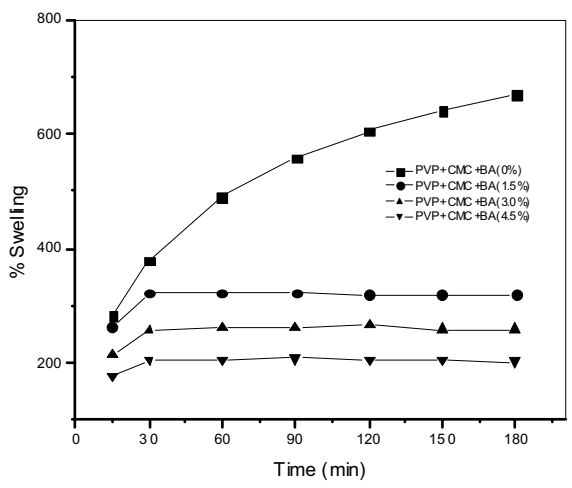

FIGURE 5. Swelling behavior of PVP-CMC-BA hydrogels in blood. 


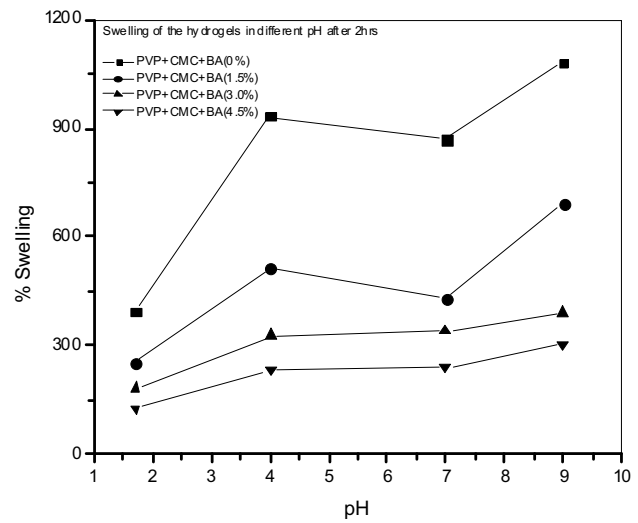

FIGURE 6. Swelling behavior of PVP-CMC-BA hydrogels in different $\mathrm{pH}$ after $2 \mathrm{hrs}$.

\section{Antimicrobial Property}

Measurement of antimicrobial properties is necessary prior to recommend the hydrogel for medical application. Figure 7 shows antibacterial effects of hydrogels on (A) Staphylococcus aureus and antifungal effects of hydrogels on (B) Candida albicans through the formation of zone of inhibition. It is clear from the images that PVP-CMC hydrogels do not have antimicrobial properties, whereas PVP-CMC-BA hydrogels are strong resistant against microbes. PVP-CMC-BA hydrogels containing $3 \%$ boric acid is shown in the figure, because incorporation of $3 \% \mathrm{BA}$ is the maximum safe limit for human body as recommended by physician. Figure 7 proves that presence of BA (3\%) is very effective to generate antimicrobial property in hydrogel.

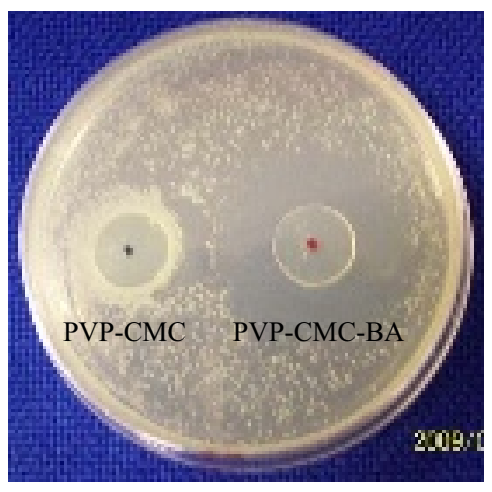

(A)

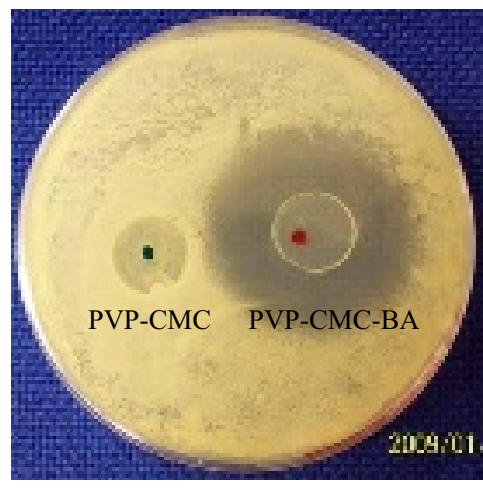

(B)

FIGURE 7. Image of antimicrobial effects of hydrogels (A) Staphylococcus aureus \& (B) Candida albicans. 


\section{CONCLUSION}

BA is enriched with antiseptic, antifungal, antibacterial and astringent properties all together and being used in medical applications like in antiseptic creams and solutions. But preparation of hydrogels with BA is new in medical field. Swelling, rheological and antimicrobial properties of PVP-CMC-BA hydrogels support for its application for wound dressing. Hydrogel with $3 \%$ BA may be optimized as an ideal wound dressing material according to its characteristic features described in the paper, and at the same time use of $3 \% \mathrm{BA}$ is the maximum permissible limit for human beings as recommended by physician.

\section{ACKNOWLEDGMENTS}

The authors are thankful to the Ministry of Education, Youth and Sports of Czech Republic (MSM 70088352101) for financial support.

\section{REFERENCES}

1. http://www.pspglobal.com/glossary-v.html (accessed April 2009).

2. L. Weng, X. Chen and W. Chen, Biomacromolecules 8, 1109-1115 (2007).

3. A. Marrtinez-Ruvalcaba, E. Chornet and D. Rodrigue, Carbohydrate Polymers 67, 586-595 (2007).

4. S. Mourtas, M. Haikou, M. Theodoropoulou, C. Tsakiroglou, and S. G. Antimisiaris, J. Coll. Interface Sci. 317, 611-619 (2008).

5. K. R. Park and Y. C. Nho, Radiat. Phys. Chem. 67, 361-365 (2003).

6. O. Z. Higa, S. O. Rogero, L. D. B. Machado, M. B. Mathor, and A. B. Lugão, Radiat. Phys. Chem. 55, 705-707 (1999).

7. N. Saha, N. Roy, T. Kitano and P. Saha, "Medicinal Value of Allicin Hydrogel Wound Cover". 25 th Annual Meeting of the Polymer Processing Society, Conference proceedings , Goa, India, 2009, p. 236.

8. N. Roy, N. Saha, T. Kitano and P. Saha, "Preparation and Characterization of A Hydrogel Based on Polyvinylpyrrolidone - Carboxymethyl Cellulose", Fifth International Conference on Polymer Modification, Degradation and Stabilization, Liege, Belgium, 2008.

9. P. Saha, N. Saha and N. Roy, "Hydrogel wound Covering" Patent filed at Czech patent office (UPV CR). File number PV 2008-306 (16 May 2008).

10. http://www.buzzle.com/articles/boric-acid.html (accessed April 2009).

11. N. Saha, N. Roy et al., "Polymeric Biomaterials Promise For Healthcare Application", International Conference on Hi-Tech Materials (ICHTM 09), Conference proceedings, IIT, Kharagpur, India, 2009 , p. 16.

12. N. Roy, N. Saha, T. Kitano and P. Saha, "Effect of Swelling on Rheological Behavior of Hydrogels", 25 th Annual Meeting of the Polymer Processing Society, Conference proceedings, Goa, India, 2009, p. 193. 
Copyright of AIP Conference Proceedings is the property of American Institute of Physics and its content may not be copied or emailed to multiple sites or posted to a listserv without the copyright holder's express written permission. However, users may print, download, or email articles for individual use. 